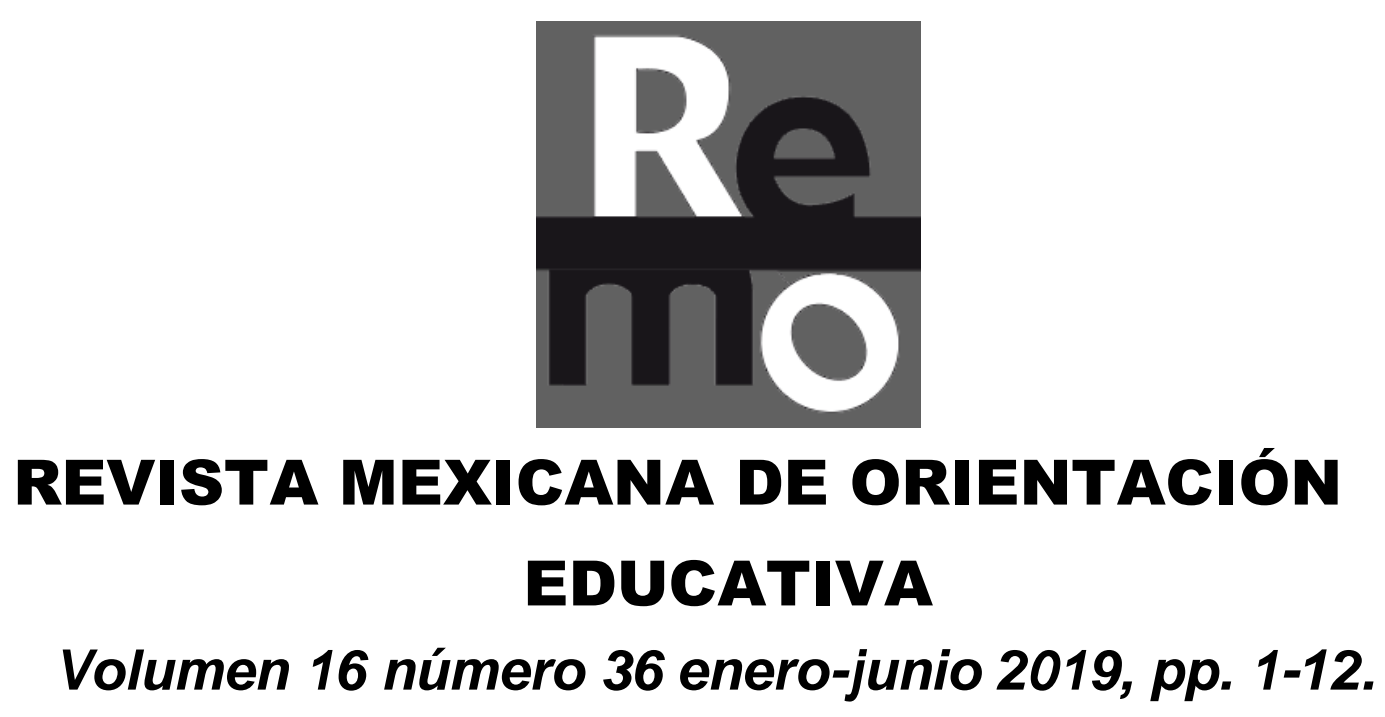

\title{
TUTORÍAS ENTRE LO IDEAL Y LO REAL: REFLEXIONES A PARTIR DE LA PRÁCTICA DOCENTE.
}

TUTORSHIP BETWEEN THE IDEAL AND THE REAL. CONSIDERATIONS FROM TEACHING PRACTICE.

\section{TUTORIAS ENTRE O IDEAL E O REAL: REFLEXÕES DA PRÁTICA DOCENTE.}

\begin{abstract}
Elsa Del Carmen Villegas Morán ${ }^{1}$, Universidad Autónoma de Baja California, evillegas@uabc.edu.mx Yazmin Vargas Gutiérrez², Universidad Autónoma de Baja California, yazmin@uabc.edu.mx Graciela Paz Alvarado ${ }^{3}$, Universidad Autónoma de Baja California, grace@uabc.edu.mx Ma. Elena Zermeño Espinosa ${ }^{4}$, Universidad Autónoma de Baja California, maelena@uabc.edu.mx
\end{abstract}

\footnotetext{
${ }^{1}$ Maestra en Comunicación Universidad de la Habana. Profesor -Investigador Universidad Autónoma de Baja California

2 Maestra en Comunicación Universidad de la Habana. Profesor -Investigador Universidad Autónoma de Baja California. Encargada de Acreditación Facultad de Ciencias Humanas.

3 Doctora en Comunicación Social Universidad de la Habana. Profesor -Investigador Universidad Autónoma de Baja California. Líder del Cuerpo Académico Procesos de Comunicación en Organizaciones e Instituciones Sociales

${ }^{4}$ Doctora en Comunicación Social Universidad de la Habana. Profesor -Investigador Universidad Autónoma de Baja California.
} 


\section{DOI: https://doi.org/10.31206/rmdo152019}

\section{Fecha de recepción: 4 de abril de 2019}

\section{Fecha de aprobación: junio de 2019}

\section{RESUMEN}

Las instituciones de educación superior (IES) en México, llevan a cabo con cierta periodicidad diversas acciones encausadas a evaluar, reestructurar o modificar sus programas de estudio; en cuya organización curricular, debe asegurar y garantizar la calidad educativa que permita a los titulados de comunicación responder profesionalmente conforme las demandas de los tiempos actuales. Para salvaguardar la calidad de los profesores universitarios, las IES han creado nuevas figuras educativas que si bien son entendidas en esencia, los catedráticos se enfrentan a una realidad distinta al momento de ponerlos en práctica, como sucede en algunos de los programas de estudios en nuestro campus universitario. Este trabajo es una reflexión que permite realizar propuestas estratégicas para el desarrollo de la actividad tutorial partiendo de consideraciones sobre la labor tutorial que se realiza actualmente dentro de la Licenciatura en Ciencias de la Comunicación de la Facultad de Ciencias Humanas, comparando con las propuestas que expresa la Asociación Nacional de Universidades e Instituciones de Educación Superior (ANUIES) sobre las tutorías y su práctica en el día a día en la labor docente universitaria.

PALABRAS CLAVE: Tutorías académica, calidad educativa, práctica docente-rezago, deserción escolar

\section{Abstract}

The higher education institutions (IES by its Spanish acronym) in Mexico, carried out, with certain periodicity, various actions prosecuted to evaluate, restructure or modify their study programs; in whose curricular organization, must ensure and guarantee the quality of education to enable the graduates respond professionally to the demands of the current times. To safeguard the teaching quality, the (IES) have created new figures that are understood in essence, nevertheless the university teachers face a different reality at the time to put them into practice, as is the case in some of the degrees in our University campus. This work is a reflection that enables strategic proposals for the development of the tutorial activity on the basis of considerations on the tutorial task that is currently within the Bachelor's Degree in Communication Sciences of the Faculty of Human Sciences comparing with the proposals which expresses the National Association of Universities and Higher Education Institutions (ANUIES by its Spanish acronym) about tutorials and his practice in the day to day work of university teaching

Key words: Academic tutoring, education quality, teaching practice, lag- dropout

RESUMO 
As As instituições de educação superior (IES, pelas suas siglas em espanhol) em México, levam a cabo com certa periodicidade diversas ações dirigidas a avaliar, estruturar e modificar seus programas de estúdio; em cuja organização curricular, deve assegurar e garantir a qualidade educativa que permita aos titulados do bacharelado em comunicação responder profissionalmente segundo as demandas dos tempos atuais. Para salvaguardar a qualidade dos professores universitários, as IES hão criado novas figuras educativas que embora são entendidas em essência, os catedráticos enfrentam-se a uma realidade distinta ao momento de põe-nos em prática, como acontece em alguns dos programas de estudo em nosso campus universitário. Este trabalho é uma reflexão que permite realizar propostas estratégicas para o desenvolvimento da atividade tutorial baseada em considerações sobre o trabalho tutorial que atualmente é feito dentro do Bacharelado em Ciências da Comunicação da Faculdade de Ciências Humanas, comparando as propostas da Associação Nacional de Universidades e Instituições de Ensino Superior (ANUIES, pelas suas siglas em espanhol) sobre as tutorias e sua prática no dia a dia no Bacharelado de comunicação.

PALAVRAS-CHAVE: Tutorias Acadêmicas, qualidade educacional, prática docente, atraso, deserção escolar

\section{INTRODUCCIÓN}

La sociedad del siglo XXI presenta una nueva dinámica sustentada fundamentalmente en el conocimiento, por lo cual las Instituciones de Educación Superior (IES) mexicanas buscan responder al reto de formar profesionistas con excelencia académica, respaldados en valores y comprometidos con su entorno por lo que requieren transformar la forma en que operan y la vinculación que guardan con la sociedad.

El Banco Mundial en su informe 2007 indica que se debe invertir en los jóvenes impactando de esta manera en la sociedad. Reconoce la necesidad de reorientar las políticas institucionales en torno a las siguientes temáticas: Oportunidades de salud y educación, capacidad de toma de decisiones, uso de la información y segundas oportunidades.

Sin embargo, según la Encuesta Nacional de la Juventud (2010) en México, el $56.15 \%$ de los jóvenes habrá dejado de estudiar al cumplir los 16 años, y en general no considera que el estudiar le permitirá obtener un trabajo mejor remunerado. Esta encuesta muestra incertidumbre del joven mexicano ante el porvenir, inclusive señalan los jóvenes, que el futuro es tan dudoso que es mejor vivir al día (49\%).

De esta manera, los jóvenes se enfrentan a un mundo en desesperanza donde consideran que no vale la pena el esfuerzo del estudio. Por otro lado, en nuestro país, solo el 5.3\% de los jóvenes estudian y trabajan; mientras que, un $22 \%$ permanecen inactivos esto se enfatiza en las mujeres (IMJUVEN, 2010). 
A pesar de las modificaciones en las políticas educativas en México, persiste el rezago social a través de el escaso ingreso de los jóvenes a la educación media y superior, o bien, el abandono provocado tanto por la falta de apoyo como por los problemas económicos y la consideración de que la educación realmente no les traerá un mejor futuro (IMJUVEN, 2010). Por el contrario, se podría decir que solo grupos y sectores poderosos de la sociedad han tenido control sobre la educación que utilizan como herramienta que refuerza las formas existentes de control y desigualdad. Ante los retos que plantea la educación del siglo XXI, las instituciones de educación superior hacen esfuerzos por superar los obstáculos que genera la sociedad mexicana.

En el año 2000, el Programa de Desarrollo Educativo generado por la administración federal señalaba como una de las líneas estratégicas de acción, la incorporación de las universidades a un sistema centrado en el aprendizaje, "caracterizado por la innovación, la flexibilidad y la movilidad estudiantil” (Programa de Desarrollo Educativo; citado por Comité de Tutorías, 2005, p. 5) para lo cual, la Facultad de Ciencias Humanas que inició en el 1993-2 con el plan de estudios flexible, implementó el sistema de tutorías como un apoyo de orientación para el estudiante.

Díaz de Cossío señaló en 2002 (citado por ANUIES, 2002, p.17) que entre los problemas más frecuentes en las instituciones de educación superior de México se encontraban: la deserción escolar definida como "el abandono que hace el alumno de los cursos o carrera a los que se ha inscrito dejando de asistir a clase y de cumplir obligaciones fijadas" y el rezago académico precisado como "el atraso en la inscripción de las asignaturas subsecuentes del plan de estudios al término de un periodo lectivo" con ello se genera, bajos índices de eficiencia terminal que se puntualiza como la relación cuantitativa entre los alumnos que ingresan y los que egresan de una misma cohorte".

La información anterior indica que de cada 100 estudiantes que iniciaron en ese año, solamente entre 50 y 60 concluían su licenciatura y 20 sólo se titularían, por lo que Asociación Nacional de Universidades e Instituciones de Educación Superior (ANUIES) consideró la actividad de tutorías como una labor esencial para abordar de frente este problema (ANUIES, 2002). Se reconoce entonces la labor tutorial como una actividad con gran potencial para reducir el problema de abandono escolar.

Apoyar a los alumnos de las IES (Instituciones de Educación Superior), con programas de tutoría y desarrollo integral, diseñados e implementados por las mismas, de suerte que una elevada proporción de ellos culmine sus estudios en el plazo previsto y logre los objetivos de formación establecidos en los planes y programas de estudio (ANUIES, 2000, p. 6)

De esta manera, la actividad de tutoría se muestra como una propuesta para solucionar problemas como el abandono escolar, con el fin de lograr un desarrollo integral que permita un óptimo aprovechamiento de la educación obtenida para su aplicación en el contexto real. 


\section{DESARROLLO}

ANUIES (2000, p. 29) considera las tutorías como:

Un proceso de acompañamiento durante la formación de los estudiantes que se concreta mediante la atención personalizada a un alumno o a un grupo reducido de alumnos por parte de académicos competentes y formados en ésta función apoyándose conceptualmente en las teorías del aprendizaje.

Se destaca dentro de esta visión, la autorrealización y desarrollo del alumno, la cual plantea que la educación debe ser centrada en la enseñanza del alumno, para ello, define que la función del maestro es la de un terapeuta, facilitador, que va a crear un clima favorable de aprendizaje, al otorgarle confianza plena durante todo el proceso de enseñanza. Así, se propone una educación más allá de los contenidos, pues plantea el aprendizaje vivencial, en el que existe un compromiso personal y pone en juego factores afectivos y cognoscitivos que facilitan el aprendizaje.

También se considera que los profesores deben implicarse en situaciones formales y no formales de aprendizaje, con sus estudiantes, así como con sus tutorados. En este sentido se rescata la importancia del aprendizaje autónomo, ya que el estudiante, en lo individual o en grupo, dirige, desarrolla y evalúa sus actividades de aprendizaje.

Este tipo de aprendizaje, aunque por sí solo implique individualidad del alumno, demanda la colaboración y apoyo de todos los miembros del grupo, de profesores, de compañeros de otros grupos o de cursos más avanzados, como lo explicó Vygotski (como se cita en Hernández, 1999) con el concepto de zona de desarrollo próximo. La tutoría implica el acompañamiento de sus tutores, para que guíen y refuercen, el proceso de aprendizaje.

El Manual de Fundamentación y Operatividad de Tutorías de la Facultad de Ciencias Humanas de la Universidad Autónoma de Baja California (Comité de Tutorías FCH, 2005) señala al tutelaje como una práctica fundamental dentro de la cual se agrupan tres objetivos básicos: primero, el desarrollo de valores, hábitos y actitudes positivas que se requieren dentro del desempeño tanto social como académico; como un segundo objetivo, señala el incremento y la probabilidad de tener éxito en el estudio y; por último, el reconocimiento de las características del plan de estudios, modalidades de estudio y acreditación para la mejor toma de decisiones (Programa de Desarrollo Educativo; citado por Comité de Tutorías, 2005, p. 10).

Sin embargo, en la realidad dentro de la Licenciatura en Ciencias de la Comunicación, las tutorías que se realizan otorgan a los alumnos información sencilla y concentrada, a través de escasos contactos, lo que provoca en ocasiones que no se llenen las necesidades y expectativas de los alumnos. De esta manera, la información otorgada puede considerarse "indebidamente dominante", pues se ve sometida a las fuerzas de producción escolar donde el egreso es un valor mucho más grande que las expectativas individuales. 
Debido a esto, el presente trabajo tiene como objetivo principal caracterizar la práctica tutorial real que se desarrolla en la Facultad de Ciencias Humanas, dependiente de la Universidad Autónoma de Baja California, se busca responder a la pregunta ¿Cuál es la práctica tutorial real que se desarrolla en la Facultad de Ciencias Humanas?

En este tenor, podemos señalar que en la dinámica de los escasos contactos y la premura que imponen los procesos de reinscripción, labor principal del tutor en la Facultad de Ciencias Humanas, el tutor llega a realizar elecciones que le corresponden al alumno. Con ello, deja muy lejos la idea inicial de Honoré (1980), quien señaló que en la tutoría debe existir una relación compartida "se trata de cultivar juntos todas las posibilidades de adquisición u expresión, de compartir la obra cultural en un esfuerzo común de compresión, de significación, de renovación, a veces de creación" (p.27).

Eso se convierte así en una situación injusta para los tutorados que son guiados en grupo, ya que inclusive algunas actividades tutoriales se imparten en conjunto al ser dirigidos hacia ciertas áreas, actividades y decisiones específicas, lo que puede provocar, la insatisfacción de las expectativas de los alumnos, factores reconocidos como generadores de la deserción escolar.

En Facultad de Ciencias Humanas, los tutores no son elegidos por los alumnos, y en el caso de tener problemas para acercarse a su tutor, ya sea por afinidad personal, académica; o bien, por problemas de horario. Los cambios son difíciles, ya que existe un protocolo y fechas establecidas que lleva a los participantes a la simulación: obtener información y apoyo de otro tutor o alumno.

La imposibilidad del alumno para elegir tutor implica posteriormente problemas, ya que es necesario que entre uno y otro exista compatibilidad y empatía; en tanto que, la diferencia de intereses profesionales y académicos, así como la falta de confianza en la interrelación entorpece considerablemente el acompañamiento tutorial.

Por su parte, ANUIES (2008) señala que para la asignación tutorial, deben equipararse los perfiles de tutores y tutorados; para ello, se debe tener en cuenta áreas de especialización del tutor y el rendimiento escolar de los alumnos; y no, de manera aleatoria anteponiendo lo administrativo como eje principal para este ejercicio, como sucede en la Facultad de Ciencias Humanas.

Es necesario reconocer previamente, ante el aumento de ingreso de estudiantes, existe una mayor heterogeneidad en ellos, características diferenciales. También es importante señalar que, a través del plan flexible y por competencias han aumentado las posibilidades de áreas y modalidades de acreditación, esto implica para el tutor manejar un gran flujo de información, para llevar a los alumnos de manera especializada conforme a sus intereses y requerimientos.

La práctica de tutorías que se realiza en la Licenciatura en Ciencias de la Comunicación (LCC) de la Facultad de Ciencias Humanas, los tutores no siempre reconocen su papel de mediador principal, se convierten más bien, en un nudo informativo donde constantemente los mensajes 
quedan estancados; o bien, ocultos a sus usuarios finales. La información oficial respecto a las tutorías, no la debería proporcionar alguna persona diferente al tutor como son: fechas y proceso de cambio de tutor, materias a elegir en otra facultad, modalidades de estudio, modalidades de acreditación, actividades académicas extraescolares, movilidad estudiantil y apoyos de las instancias universitarias; por lo cual, surgen espontáneamente alumnos de semestres avanzados quienes llegan a otorgar guías de acción basadas en sus experiencias personales.

De igual manera, dentro de estos escasos contactos que nacen específicamente del proceso de reinscripción, el tutor en la LCC obtiene información importante de los alumnos que después no hace llegar a las instancias correspondientes, ya que no existen los procesos establecidos para ello.

Es de notarse que tampoco la información administrativa circula adecuadamente para los tutores; cambios de salón, grupo o maestro. De manera similar, la información académica a la que el tutor debe tener acceso para realizar este proceso de acompañamiento no está disponible directamente y tiene en su caso, que solicitarla a otras instancias.

Como es sabido, el conocer la trayectoria escolar de los alumnos permite al tutor identificar fortalezas y debilidades de ellos, esta información permitir al tutor, de manera más objetiva, crear planes y estrategias adecuadas a cada alumno; por lo tanto, al no tener información el tutor depende totalmente de la información escolar que el estudiante pueda recolectar y otorgar antes de llegar a la entrevista.

Por otro lado, los tutores consideran que el alumno tiene otras vías para obtener la información que requiere, o bien, para el tutor, el alumno no requiere información o no está interesado; al no ser siempre atendidas las convocatorias a reuniones con alumnos.

La Universidad Autónoma de Baja California (UABC, 2011, p. 22) fija que la tutoría la ejerce un docente "quien cuenta con la formación, las habilidades y aptitudes necesarias". Sin embargo, es importante notar que en la Facultad de Ciencias Humanas la capacitación para ser tutor, no es una condición necesaria, ya que no existe una forma de acreditación de maestros como tutores y si bien existe un manual de tutorías aprobado por el Consejo Técnico de la unidad académica (diciembre, 2004), este no es conocido por quienes ejercen esta labor.

Debido a que los tutores del programa educativo en comunicación no han sido capacitados en tópicos como: integración a la vida universitaria, motivación, proyectos de vida, auto evaluación del aprendizaje, técnicas y estrategias de aprendizaje y administración del tiempo, los temas no son abordados dentro de la tutoría y de presentar algún problema los estudiantes son enviados al departamento psicopedagógico.

Dicha realidad se muestra muy lejana a la propuesta de Alcántara Santuario (1990, p. 51) donde señala que: 
La tutoría es una forma de atención educativa donde el profesor brinda su apoyo al estudiante de manera sistemática, por medio de la estructuración de objetivos, programas de organización por áreas, técnicas de enseñanza apropiadas conforme a ciertos criterios y mecanismos de monitoreo y control entre otros.

Si bien existe un sistema de seguimiento tutorial no es posible constatar si desde la implementación del programa de tutorías en el periodo 1993-2, se ha reducido el abandono escolar o el rezago educativo. Sin estos datos, el tutor no tiene las herramientas para desarrollar de manera adecuada su actividad como lo describe la ANUIES (2008) al señalar la importancia de establecer métodos e instrumentos que permitan conocer el impacto de las tutorías a partir del rendimiento académico.

Sin embargo, la Universidad Autónoma de Baja California emite un reporte de la evaluación tutorial a partir de las consideraciones realizadas voluntariamente por estudiantes, este reporte, no contribuye en la práctica a mejorar las acciones tutoriales ya que no evalúa de manera profunda y los resultados obtenidos allí no generan ningún tipo de seguimiento (tabla 1).

Tabla 1. Reporte de Evaluación Tutorial

\begin{tabular}{|l|l|}
\hline \multicolumn{1}{|c|}{ Preguntas } & \multicolumn{1}{c|}{ Indicador } \\
\hline Encuentro a mi tutor en el horario asignado & Cumplimiento del horario \\
\hline $\begin{array}{l}\text { La relación con mi tutor es cordial y de respeto } \\
\text { El tutor me orienta en mi elección de las unidades y actividades de } \\
\text { aprendizaje que conforman mi carga académica }\end{array}$ & $\begin{array}{l}\text { Trato con los estudiantes } \\
\text { Orientación académica }\end{array}$ \\
\hline $\begin{array}{l}\text { El tutor me orienta en las instancias adecuadas cuando mis dudas } \\
\text { corresponden a otra área }\end{array}$ & Orientación académica \\
\hline $\begin{array}{l}\text { Acudo de manera puntual a las citas con mi tutor } \\
\text { Realizo las actividades que me asigna mi tutor }\end{array}$ & $\begin{array}{l}\text { Asistencia } \\
\text { pumplimiento tareas por }\end{array}$ \\
\hline $\begin{array}{l}\text { Me parece útil y necesario este programa de tutorías } \\
\text { Ingreso con facilidad al sistema }\end{array}$ & Percepción utilidad \\
\hline $\begin{array}{l}\text { El sistema de Tutorías (web) brinda información que me permite } \\
\text { construir mi proyecto académico profesional }\end{array}$ & Utilidad del sistema \\
\hline
\end{tabular}

Elaboración propia a partir de datos obtenidos en el Sistema Institucional de Tutorías UABC

Dentro del sistema de evaluación solamente cuatro de las diez preguntas están orientadas a evaluar el funcionamiento real de la actividad tutorial realizada por cada académico, mientras que otras dos se refieren a una autoevaluación de los estudiantes y las restantes a evaluar el funcionamiento del sitio web en general. Es decir, aportan realmente poco al conocimiento sobre la actividad tutorial que se realiza. 
Si bien reconocemos que tanto el abandono escolar como el rezago académico son problemas multicausales y que inciden en ellos factores como: características individuales de los estudiantes, problemas económicos, sociales y familiares; así como la desventaja educativa y cultural. Es innegable el papel que podrían jugar las tutorías, por lo que el monitoreo de la actividad tutorial y la posterior publicación de los datos permitirá identificar el impacto real de las tutorías y; en su caso, introducir estrategias que permitan a través del proceso tutorial reducir los índices de deserción y rezago escolar; con ello, incrementar la eficiencia terminal.

Otro aspecto negativo por la inexistencia de mecanismos de evaluación tutorial en la Licenciatura en comunicación, es que el profesor-tutor no se ve obligado a cumplir con las labores que competen a la tutela y en algunos casos ceden esta actividad a otros profesores, o bien, al coordinador general de tutorías o al coordinador de carrera.

No obstante, dicha ausencia de compromiso académico, el profesor-tutor conserva su nombramiento que administrativamente le favorece para lograr el perfil (PROMEP), Programa de Mejoramiento del Profesorado mediante el cual, no solo le permite obtener beneficios económicos, sino conservar el reconocimiento al perfil deseable que se obtiene como resultado de las múltiples actividades inherentes a la formación académica, investigación, productividad, gestión y docencia que debe de realizar un Profesor de Tiempo Completo (PTC) de la Universidad Autónoma de Baja California y por ende, de la Licenciatura en comunicación.

En cuanto a la formación, productividad e investigación académica los PTC de la LCC, el 70\% tienen grado de doctor y un $30 \%$ se encuentran actualmente realizando estudios doctorales. Asisten y participan en congresos nacionales e internacionales. En investigación, el $90 \%$ de los PTC forman parte de Cuerpos Académicos, ya sea en calidad de miembros o colaboradores. Además, participan en proyectos de investigación que se encuentran registrados ante la Coordinación de Posgrado e Investigación de la universidad, y se encuentran en constante y permanente producción de artículos académicos como resultados de esta actividad.

Respecto a la gestión, los docentes participan en las labores de coordinación en distintas áreas académico-administrativas que involucran tanto a la formación escolar como al acompañamiento tutorial de los alumnos del programa.

De la docencia, las horas clase son una labor sustantiva del profesor de tiempo completo de la Licenciatura en Ciencias de la Comunicación; es por ello, que se tienen asignadas desde 12 horas semana mes, como mínimo, hasta 20 horas semana mes como máximo. Asimismo, las horas que se imparten en posgrados que regularmente oscilan entre tres a seis horas semana mes (tabla 2).

Tabla 2 Distribución carga académica Profesores de Tiempo Completo de la Licenciatura en Ciencias de la Comunicación 


\begin{tabular}{|l|l|l|}
\hline \multicolumn{1}{|c|}{13 PROFESORES T/C } & \multicolumn{1}{|c|}{$\begin{array}{c}\text { NÚMERO DE HORAS A LA } \\
\text { SEMANA }\end{array}$} & $\begin{array}{c}\text { PORCENTAJE DE HORAS } \\
\text { PARA LA ACTIVIDAD }\end{array}$ \\
\hline HORAS CLASE & 160 & $30 \%$ \\
\hline HORAS INVESTIGACIÓN & 58 & $11.1 \%$ \\
\hline TUTORÍAS & 102 & $19 \%$ \\
\hline COORDINACIÓN & 58 & $11.1 \%$ \\
\hline $\begin{array}{l}\text { ASEGURAMIENTO DE LA } \\
\text { CALIDAD }\end{array}$ & 22 & $4.2 \%$ \\
\hline COMISIÓN A OTRA UNIDAD & 100 & $19 \%$ \\
\hline LABORATORIOS & 18 & $3.4 \%$ \\
\hline
\end{tabular}

Fuente: elaboración propia

Por lo tanto, las tutorías académicas como actividad sustantiva, no son tal; en tanto que en la licenciatura no es sino una actividad más, a la que el profesor debe atender a un número de alumnos que varía desde cinco hasta 70; que aunado a la falta de interés de parte de algunos estudiantes, no favorece para llevar a cabo la práctica tutorial ya que existe un elevado número de estudiantes que no corresponde con la reducida cantidad de tutores capacitados. Esto aunado a la falta de precisión en la definición de las tareas del tutor la labor de tutorías (ANUIES, 2008).

Otro elemento a observar, es que la ANUIES (2008) considera que los alumnos de nuevo ingreso son más vulnerables en términos de deserción; particularmente para el objeto de análisis de este. En la Facultad de Ciencias Humanas, en esta etapa que integra a todos los alumnos de nuevo ingreso en un área interdisciplinaria que denomina Tronco Común, la tutoría académica se imparte mayormente de manera grupal y no personal como el acompañamiento tutorial indica.

Es de reconocer que si bien la planta académica y la comunidad universitaria en general se ve beneficiada, cuando un PTC logra el grado de doctor, esto no está necesariamente relacionado con un efectivo desempeño como tutor ya que en muchos casos tendrá menos tiempo real para dedicar a esta actividad. Además de que los conocimientos científicos no serán suficientes para realizar la actividad de tutorías, que como se ha dicho, requiere también experiencia y formación pedagógica.

Finalmente, la tabla 3 presenta un comparativo de las tutorías ideales propuestas por ANUIES y las tutorías reales de la Licenciatura en comunicación de la Facultad de Ciencias Humanas.

Tabla 3. Análisis de Práctiva Tutorial

\begin{tabular}{|l|l|}
\hline \multicolumn{2}{|c|}{ IDEAL } \\
\hline \multicolumn{2}{|c|}{ ASIGNACIÓN } \\
\hline Selectiva identificando perfiles y necesidades. & $\begin{array}{l}\text { Aleatoria, basada en situaciones } \\
\text { administrativas y necesidades de } \\
\text { puntuación del profesor. }\end{array}$ \\
\hline \\
IMPARTICIÓN
\end{tabular}




\begin{tabular}{|c|c|}
\hline Sólo profesores de tiempo completo. & $\begin{array}{l}\text { Profesores de tiempo completo, analistas, } \\
\text { profesores de asignatura e investigadores }\end{array}$ \\
\hline Acompañamiento durante toda la carrera. & $\begin{array}{l}\text { Apoyo semestral al momento de la } \\
\text { reinscripción. }\end{array}$ \\
\hline Se requiere información del tutorado. & $\begin{array}{l}\text { No siempre es posible acceder a la } \\
\text { información del tutorado. }\end{array}$ \\
\hline Número reducido de estudiantes. & Número máximo de estudiantes. \\
\hline \multicolumn{2}{|c|}{ PREPARACIÓN } \\
\hline $\begin{array}{l}\text { Sistemática a través de cursos de capacitación y } \\
\text { habilitación. }\end{array}$ & Voluntarias a través de cursos esporádicos. \\
\hline \multicolumn{2}{|c|}{ EVALUACIÓN } \\
\hline $\begin{array}{l}\text { Permanente que permita la modificación de las } \\
\text { acciones. }\end{array}$ & $\begin{array}{l}\text { Semestralmente por los estudiantes } \\
\text { mediante sistema Web como requisito para } \\
\text { realizar el proceso de reinscripción al } \\
\text { siguiente periodo escolar. }\end{array}$ \\
\hline
\end{tabular}

\section{CONCLUSIONES Y PROPUESTAS}

Debido a que las tutorías implican una labor no sólo informativa sino formativa más allá de lo académico hasta lograr ser un apoyo al desenvolvimiento de la persona en todas sus dimensiones, podemos concluir que dentro de la Licenciatura en Ciencias de la Comunicación de la Facultad de Ciencias Humanas, la actividad tutorial no cumple a cabalidad con los indicadores de asignación impartición y capacitación propuestos por la ANUIES. Por otro lado, la evaluación se cumple de manera permanente sin embargo no se enlaza con elementos de evaluación importantes como la permanencia y el egreso de los estudiantes.

Como propuestas finales estas autoras consideran que es necesario reconocer que el ser formador-tutor no es espontáneo, sino que al igual que otras actividades solicitadas al catedrático actual como la investigación y la docencia requieren ser aprendida y cultivada.

De igual manera, es determinante mostrar el papel del alumno en el proceso, aplicación y desarrollo de su vida académica, por lo que en la labor tutorial se debe evitar tanto el dominio como la dependencia, en la que el docente debe ser capacitado para evitar el papel de un sujeto dador de la verdad y alumnos como receptores dependientes sin necesidades e intereses propios.

El alumno debe ser visto como un interlocutor activo con capacidad para generar respuestas de acuerdo a sus necesidades e inquietudes. Es importante que se reconozca a los alumnos como seres arraigados a una vida cotidiana que los une a idiosincrasias, mentalidades y actitudes creativas y productivas que habrán de ser sopesadas y aprovechadas para el mejor desarrollo académico.

Por lo cual se deben plantear y gestar acciones de comunicación que influyan en la implementación de acciones para el mejoramiento de los estudiantes, y evitar la parálisis social que impida la innovación y la creatividad. Por otra parte, deben describirse con mayor claridad 
las estrategias de comunicación a seguir dentro de la labor tutorial y reconocer a través de ello las necesidades de los alumnos.

Por último, es necesario recordar que la actividad tutorial permite promover identidades sociales, culturales, individuales en pos de mejorar las posibilidades de desempeño de los alumnos tanto al interior del ámbito académico como en el profesional, para lo cual es necesario la regulación y el establecimiento de programas específicos más allá del proceso de reinscripción.

\section{REFERENCIAS}

Alcántara, A. (julio-diciembre 1990). Consideraciones sobre la tutoría en la docencia universitaria, revista Perfiles Educativos, 13(49-50), 51-55.

Asociación Nacional de Universidades e instituciones de Educación superior. (2000), Programas institucionales de tutoría: una propuesta para su organización y funcionamiento en las IES, México: ANUIES.

Asociación Nacional de Universidades e instituciones de Educación superior. (2002). Programas Institucionales de Tutoría. Una propuesta de la ANUIES para su organización y funcionamiento en las instituciones de educación superior. México: ANUIES.

Asociación Nacional de Universidades e instituciones de Educación superior. (2008). El papel del profesor tutor en el marco de la nueva dinámica institucional. México: ANUIES.

Comité de Tutorías FCH. (2005). Manual de Fundamentación y Operatividad de Tutorías de la Facultad de Ciencias Humanas de la Universidad Autónoma de Baja California. Mexicali: UABC.

Hernández, G. (1999). La zona de desarrollo próximo. Comentarios en torno a su uso en los contextos escolares. Perfiles Educativos, (86), 1-32. Recuperado de https://mcesuvaq.files.wordpress.com/2013/08/15-la-zona-de-desarrolloprc3b3ximo.pdf.

Honoré, B. (1980). Para una teoría de la formación. Dinámica de la formatividad. Madrid: Narcea.

Instituto Mexicano de la Juventud. (2010). Investigación. México:IMJUVEN. Recuperado de http//:www.cendoc.imjuventud.gob.mx/investigación/.

Universidad Autónoma de Baja California (2011). Agenda Universitaria. México: ABC.

Universidad Autónoma de Baja California (2011). Población Estudiantil Licenciatura y Posgrado, Recuperado de

http://csege.uabc.mx/index.php?option=com jdownloads\&ltemid=74\&task=viewcategory\&catid $\underline{=9}$. 